\title{
Cervical spondylosis causing vertebrobasilar insufficiency: a surgical treatment
}

\author{
DONALD R. SMITH, GARY D. VANDERARK, AND LUDWIG G. KEMPE \\ From the Department of Surgery, Neurosurgery Service, Walter Reed General Hospital, Washington, D.C., $\stackrel{\overrightarrow{\bar{S}}}{\vec{\theta}}$ \\ U.S.A.
}

SUMMARY Although the most common aetiology of transient vertebrobasilar insufficiency is $\stackrel{\mathbb{Q}}{\varrho}$ atherosclerosis, a similar syndrome may occasionally be produced by cervical osteophytes. The $\tilde{\omega}$ possibility of such a remedial lesion makes further investigation mandatory in such patients- $\overrightarrow{0}$ especially if symptoms are associated with sudden movements of the head or neck. When vertebral $\overrightarrow{\vec{H}}$ compression results from osteophytes, it can be easily relieved by a minor modification of the usual $\omega_{\sigma}^{\omega}$ anterior cervical fusion technique. This method has proved to be quite efficacious in two patients whose case histories are reported.

That transient or even permanent cerebrovascular insufficiency may be produced by extracranial lesions is now a well-recognized fact. The most common aetiology is that of intraluminal atheroma producing microemboli and decreased flow volume in the carotid or vertebrobasilar systems. When such symptoms as syncope, vertigo, tinnitus, or nausea occur associated with head movements, the area of primary suspicion should be the cervical portion of the vertebral artery.

Such symptoms can arise secondary to extrinsic compression of the vertebral artery-most commonly by cervical osteophytes in the cervical spineand this has been previously documented by several investigators (Hutchinson and Yates, 1956; Lewis and Coburn, 1956; Tatlow and Bammer, 1957; Hardin, Williamson, and Steegman, 1960; Gortvai, 1964; Bakay and Leslie, 1965; Labauge, Thévenet, Crouzet, and Nivolas, 1967; Nagashima, 1969). Successful therapy of such lesions has been sporadic and generally unsatisfactory. We have recently encountered two such patients and successfully treated these by removal of osteophytes and vertebral artery decompression. This was accomplished by minor modifications and extension of the anterior discectomy approach of Cloward (1958). This method, as originally described, has been widely used for routine treatment of cervical spondylosis productive of radiculopathy or myelopathy.

This approach has previously been employed for relief of vertebral artery compression (Bakay and Leslie, 1965). The minor modifications necessary for this purpose have proved so simple and efficacious $\infty$ that we suggest it as the treatment of choice.

\section{OPERATIVE TECHNIQUE}

The standard anterior vertebral approach w followed essentially as originally presented $\overrightarrow{0}$ Cloward (1958). The essential points of this methot will be re-emphasized. The skin incision is in the right lateral cervical area extending from the midline to the middle of the sternocleidomastoid muscle. After opening the skin and platysma muscle, the cervical fascia is opened just medial to the sternocleidomastoid muscle. This muscle is then reflected laterally along with the carotid sheath and its contents. The trachea and oesophagus are retracted medially to expose the anterior vertebral bodies with the overlying longus colli muscles. These are split in the midline and retracted laterally to expose the anterior surface of the vertebral bodies and their interspaces. These muscles, and the anterior longitudinal ligament, are stripped quite far laterally to expose the entire interspace for visualization during 8 the later osteophyte removal. The Cloward selfretaining retractors are used to spread the longus $ᄋ$ colli muscles and maintain exposure. The anterior part of the annulus is then excised by sharp dissection-again care must be taken that this is carried far laterally. At this stage in the procedure, however, no attempt is made totally to expose or remove the lateral osteophytes. The soft nucleus pulposus and cartilage plates are removed by appropriate curettes 
and rongeurs. The Cloward drill and guard are then centred over the intervertebral space and advanced until the posterior longitudinal ligament is encountered. We prefer to remove this ligament and visualize the underlying dura mater, but this is not essential when the procedure is only for vertebral artery decompression. Beginning at the posterior aspect of the drill opening, it is then quite easy to carry the bony removal laterally by means of curettes and fine punch rongeurs. The latter are used very sparingly and only under direct vision for fear of lacerating the vertebral artery. The curette is felt to be much safer and in experienced hands is a more effective tool. It is a very simple matter to continue this removal of bone and osteophyte laterally until the vertebral artery is exposed (Fig. 1). Once the vessel has been visualized, the wide removal of the bony spur may be carried out without further medial or anterior bony removal. This latter should be specifically avoided as it will result in a poorly seated bone dowel.

After the bony removal, the artery can be observed to lie quite free without compression and may even bulge into the evacuated interspace. When this has been accomplished, the previously prepared dowel of autogenous iliac bone is impacted into the drill opening so that fusion will occur. The wound is approximated in anatomical layers. The patient may be allowed up in a collar or brace postoperatively just as with any anterior fusion. It has, however, been our policy to immobilize these

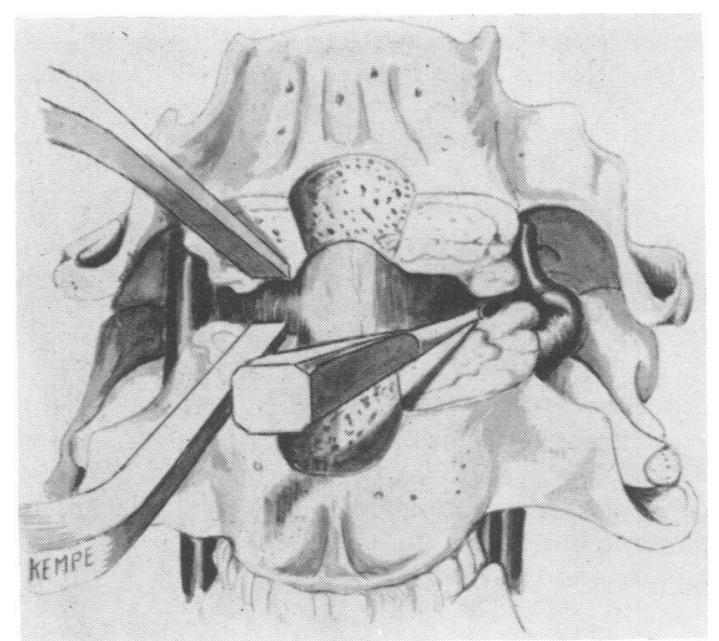

FIG. 1. Drawing to show removal of the osteophytes adjacent to the vertebral artery. The width of the anterior intervertebral space is exaggerated in the drawing to allow better display of the deep structures. patients, as we do other routine anterior cervical fusion patients, in a plaster Minerva jacket for six weeks.

\section{CASE 1}

This 51 year old woman was involved in a car accident 14 months before examination. She sustained a flexionhyperextension injury of the neck. After this accident, she had complained of headaches, loss of balance, and brief episodes of syncope. In her work as a librarian, syncope or sudden falling would frequently be precipitated when her neck was hyperextended to view the upper shelves.

Examination revealed the carotid and subclavian pulses to be normal and no bruits were present over the neck or upper chest. Neurological examination was entirely normal. Gradual hyperextension of the neck would induce dysconjugate eye movements and further provocative correlation was not attempted. Radiographs of the cervical spine revealed large lateral and posterior osteophytes at the C5-6 vertebral level. Angiograms demonstrated lateral displacement of the vertebral arteries bilaterally, but to a much greater extent on the left side (Fig. 2).

The anterior approach as described above was used to remove the C5-6 intervertebral disc and the bilateral osteophytes. Great care was taken to remove the lateral bony protrusions to expose and totally decompress the vertebral artery bilaterally. The usual interbody fusion utilizing an autogenous iliac bone dowel was performed. Routine postoperative immobilization in a plaster jacket was carried out for six weeks. After removal of the plaster, the angiograms were repeated (Fig. 3) and revealed a completely normal course of the vertebral artery. When repeatedly subjected to hyperextension of the cervical spine, the preoperative symptoms could not be reproduced.

CASE 2

This 53 year old man had complained of intermittent neck and left arm pain for nine years. For one year before admission he had experienced occasional episodes of vertigo and falling associated with sudden head turning or on looking upward. During this interval, he had also noted intermittent numbness over the left side of the face.

Examination revealed hypaesthesia in the left sixth cervical dermatome. There was decreased strength in the left biceps muscle. All deep tendon reflexes were within normal limits and symmetrical, with the exception of the left biceps reflex which was absent. The carotid and subclavian pulses were palpable and normal and there were no bruits present. Radiographs of the cervical spine revealed large lateral osteophytes at both the C4-C5 and the C5-C6 intervertebral spaces. There was a congenital fusion of the sixth and seventh cervical vertebrae. Myelography confirmed the lateral defects at both levels associated with the previously noted osteophytes. These defects were thought to be indicative of neural foraminal compression at the involved levels. Angiography revealed 

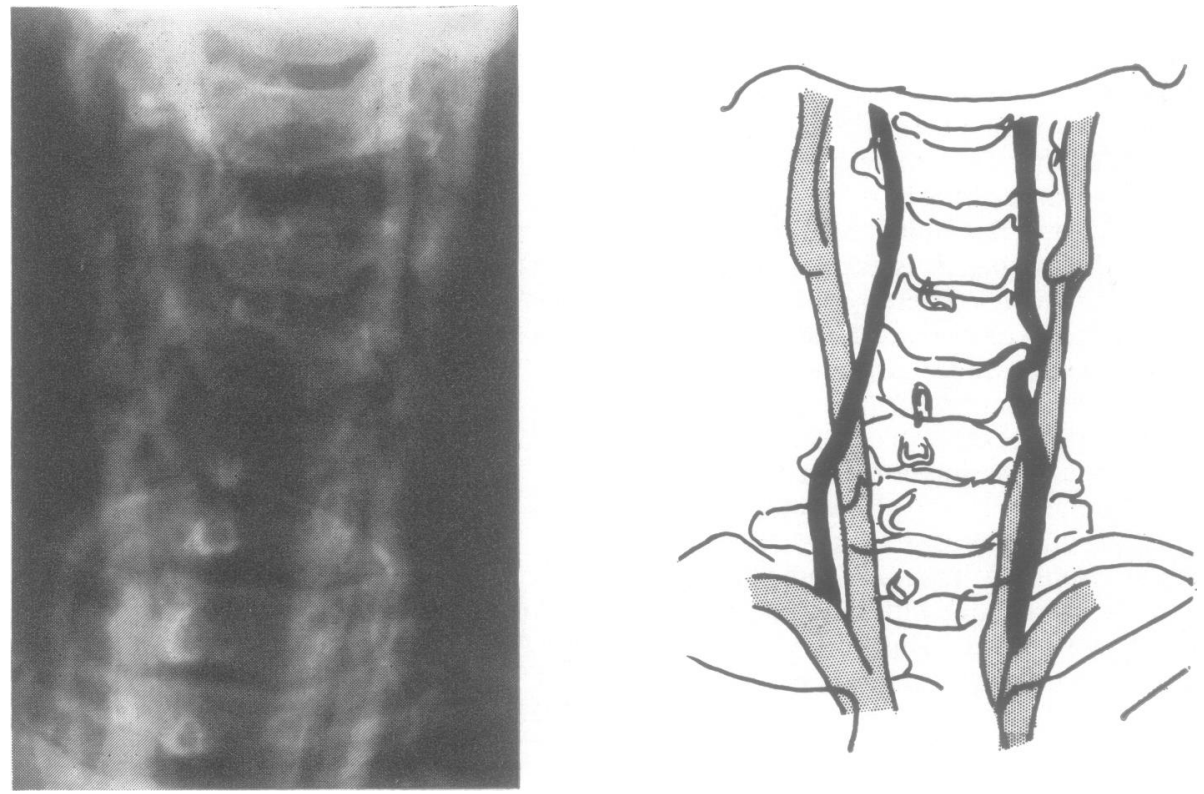

FIG. 2. Case 1. Preoperative angiogram to show the marked displacement of the left vertebral artery at one level.

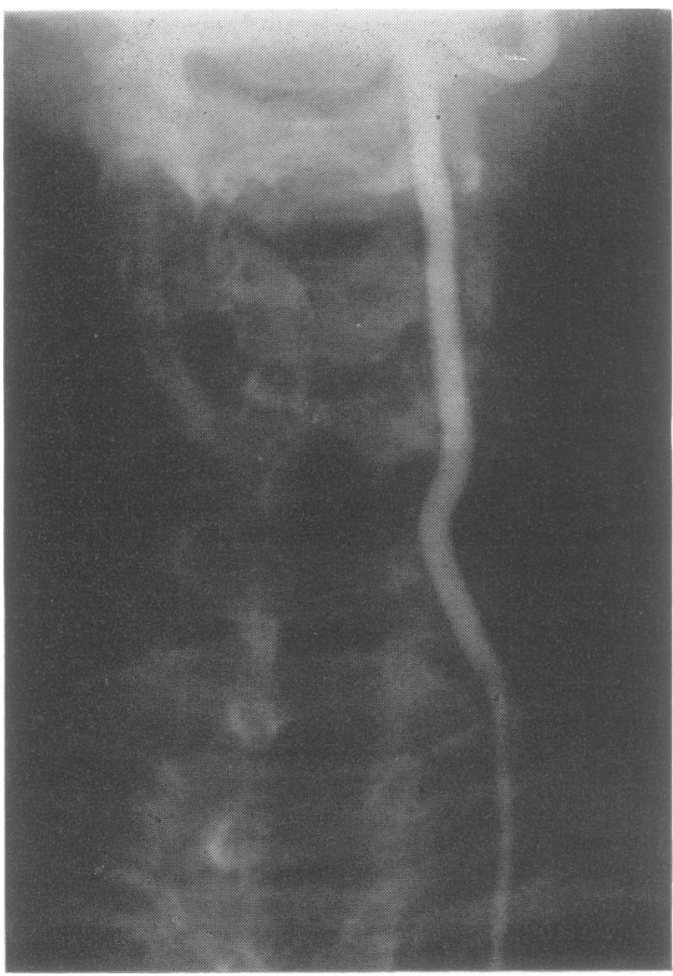

marked bilateral displacement of the vertebral arterie by the osteophytes (Fig. 4).

Bilateral two-level foraminotomy and removal of the lateral osteophytes adjacent to the vertebral artery was carried out by the anterior approach as previously deำ scribed. Postoperative angiograms revealed the vertebral displacement to be relieved (Fig. 5). Postoperatively the patient has returned to full activities. His symptoms of vertigo, dizziness, facial numbness, and falling have been completely relieved even when subjected to extremes of cervical motion during examination.

\section{DISCUSSION}

Ischaemia of the central nervous system with either permanent or transient effects is now widely recognized to be often the result of extracranial pathology. By far the most common cause is advancing atherosclerosis with plaques in the major vessels. Atheroma there may produce problems either by embolization of thrombotic material or by luminal narrowing. Although much more unusual, it is becoming increasingly obvious that ischaemic symptoms may $\mathrm{O}$ result from extravascular pathology.

FIG. 3. Case 1. Postoperative selective vertebral angiogram. The artery can be seen to deviate medially into the surgical bony defect. 

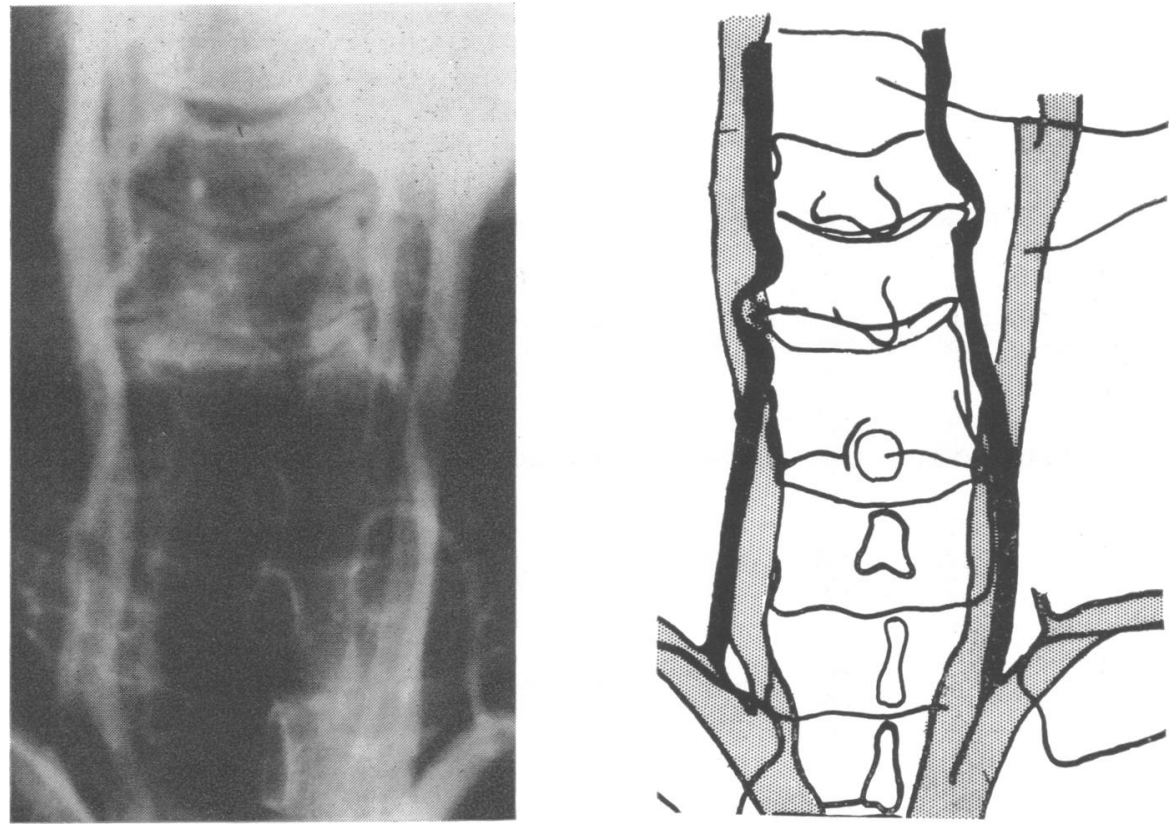

FIG. 4. Case 2. Preoperative angiogram showing vertebral artery displacement at two levels.
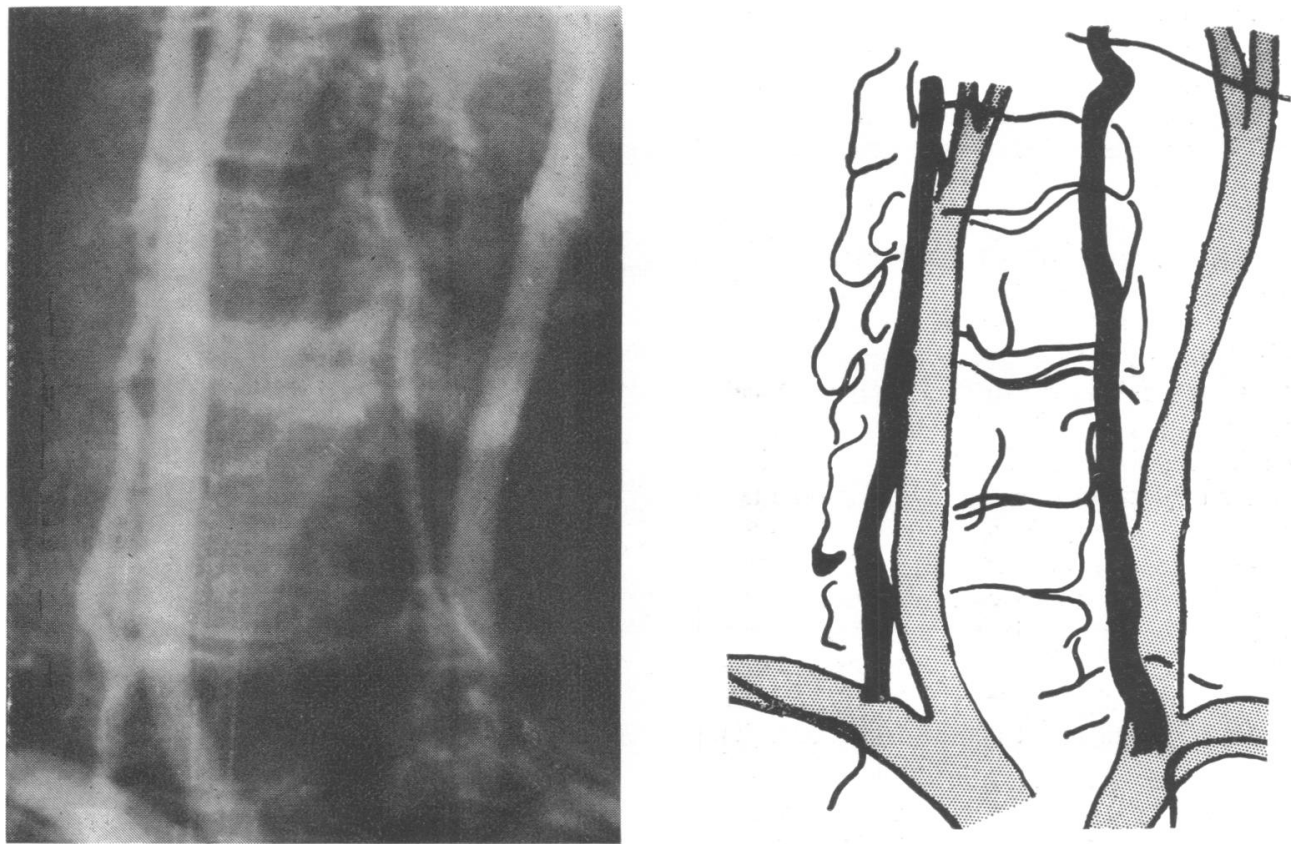

FIG. 5. Case 2. Postoperative angiogram. Both vertebral arteries take a normal course. 
Hutchinson and Yates (1956), while reviewing a large series of necropsy material with arteriography of the vertebrobasilar system, noted several patients in which the cervical portion of the vertebral artery was compressed by osteophytes. The theory was proposed that these bony spurs might play a role in obstruction and that this could occur with certain movements of the cervical spine. The vertebral arteries were also inspected at necropsy in patients who had antemortem clinical symptoms of vertigo and syncope associated with head movements (Tatlow and Bammer, 1957). Most of these patients demonstrated significant atherosclerotic involvement, but one patient was noted to have partial obstruction at the level of an osteophyte. Schneider and Crosby (1959) have described various pathological changes in the upper spinal cord and brain-stem after acute cervical trauma. These changes were felt to be on a vascular rather than a direct traumatic basis secondary to the acute vertebral artery occlusion.

Once recognized as an aetiological lesion, the osteophytes have been dealt with operatively. Thus far, all reported cases of surgical correction, with a single exception (Bakay and Leslie, 1965), have been carried out by a lateral or anterolateral approach and direct attack on the osteophytes adjacent to the vertebral artery (Radner, 1951; Hardin et al., 1960; Gortvai, 1964). The dental burr or curette was used to remove the spurs as well as a portion of the lateral vertebral body as needed for visualization. This method has reportedly been quite satisfactory in the few patients to which it has been applied.

Our familiarity with the anterior approach (Cloward, 1958; Rosomoff and Rossman, 1966) used in the routine treatment of cervical spondylosis and disc disease led to an adaptation of this method. There are other apparent advantages in this method. The most obvious is that patients developing lateral osteophytes of sufficient degree to involve the vertebral circulation may also manifest a significant incidence of cervical nerve root compression on a spondylotic basis. This radiculopathy may also be clinically evident (case no. 2). In this situation, it is a simple matter to decompress the neural elements by complete removal of the osteophytes while freeing the vertebral arteries. In addition, the establishment of a stable fusion at the involved intervertebral space may play a significant role in the relief of vascular symptoms. Although this may be unnecessary when the osteophytes are radically removed, this adds an extra degree of certainty to the procedure. The absence of further movement at this level may also prevent the future regrowth of osteophytes.

The very satisfying results in these two patients on would indicate that such treatment offers excellent clinical relief of vascular symptoms. Although this 0 remains an unusual cause for vertebrobasilar insufficiency, the ease with which it can be corrected $\stackrel{\circ}{\stackrel{9}{?}}$. would demand constant vigilance. This evaluation $\stackrel{\overrightarrow{0}}{\vec{*}}$ should include at least radiographs of the cervical $\stackrel{2}{-}$ spine in all patients presenting with these symptoms $\overrightarrow{\vec{S}}$ of vertebrobasilar insufficiency, especially if a history is elicited of aggravation by head movement. 등 When osteophytic spurs are seen, angiography is $\frac{\bar{\sigma}}{\bar{c}}$. indicated. In this manner, a small group of patients $\vec{\nabla}$ will be discovered in whom simple decompression $\cong$ will result in total relief of symptoms and return to के normal activity.

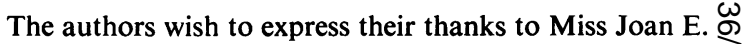
Sitman for her assistance in preparing this manuscript.

REFERENCES

Bakay, L., Leslie, E. V. (1965). Surgical treatment of vertebrâ artery insufficiency caused by cervical spondylosis. Neurosurg., 23, 596-602.

Cloward, R. B. (1958). The anterior approach for removit of ruptured cervical disks. J. Neurosurg., 15, 602-617. Gortvai, P. (1964). Insufficiency of vertebral artery treated by decompression of its cervical part. Brit. med. J., 2, 233-239

Hardin, C. A., Williamson, W. P., Steegmann, A. T. (1960 Vertebral artery insufficiency produced by cervical oste- $\overrightarrow{0}$ arthritic spurs. Neurology (Minneap.), 10, 855-858.

Hutchinson, E. C., and Yates, P. O. (1956). The cervical . portion of the vertebral artery: A clinico-pathological study. Brain, 79, 319-331.

Labauge, R., Thévenet, A., Crouzet, G., and Nivolas, M. (1967). Les insuffisances vertébro-basilaires d'incidence $\overline{\bar{O}}$ chirurgicale (à propos de 87 malades opérés). Rev. Neurol., ڤે 117, 373-389.

Lewis, R. C., and Coburn, D. F. (1956). The vertebral artery: its role in upper cervical and head pain. Missouri Med., $\overrightarrow{\bar{D}}$ 53, 1059-1063.

Nagashima, C. (1969). Surgical treatment of vertebro-basilar insufficiency due to cervical spondylosis. Brain Nerve (Tokyo), 21, 1100-1111.

Radner, S. (1951). Vertebral angiography by catheterization. A new method employed in 221 cases. Acta radiol., Suppl. No. 87, 33-34.

Rosomoff, H. L., and Rossmann, F. (1966). Treatment of $\frac{0}{3}$ cervical spondylosis by anterior cervical diskectomy and fusion. Arch. Neurol. (Chic.), 14, 392-398.

Schneider, R. C., and Crosby, E. C. (1959). Vascular insufficiency of brain stem and spinal cord in spinal trauma. Neurology (Minneap.), 9, 643-656.

Tatlow, W. F. T., and Bammer, H. G. (1957). Syndrome of $D$ vertebral artery compression. Neurology (Minneap.), 7. 331-340. 